\title{
ANNIHILATING FIELDS OF STANDARD MODULES FOR AFFINE LIE ALGEBRAS
}

\author{
JULIUS BORCEA
}

\begin{abstract}
Given an affine Kac-Moody Lie algebra $\tilde{\mathfrak{g}}[\sigma]$ of arbitrary type, we determine certain minimal sets of annihilating fields of standard $\tilde{\mathfrak{g}}[\sigma]$-modules. We then use these sets in order to obtain a characterization of standard $\tilde{\mathfrak{g}}[\sigma]$ modules in terms of irreducible loop $\tilde{\mathfrak{g}}[\sigma]$-modules, which proves to be a useful tool for combinatorial constructions of bases for standard $\tilde{\mathfrak{g}}[\sigma]$-modules.
\end{abstract}

\section{INTRODUCTION}

It is a well-known fact that suitably specialized characters of standard modules for affine Lie algebras can be expressed as certain infinite products. In turn, these products may be interpreted as generating functions of partition functions for colored partitions defined by congruence conditions (cf. An]). Such arguments have revealed the connections existing between the character theory of standard modules for affine Lie algebras and (the product side of) partition identities of RogersRamanujan type (cf. [LM]). It was therefore natural to ask whether these identities could be understood through representation theory as manifestations of a new algebraic structure that would lead to bases (of the corresponding modules) which would be parametrized by (colored partitions satisfying) difference conditions. This question has been answered in the affirmative in a range of cases - including the original Rogers-Ramanujan identities - by Lepowsky and Wilson ([LW1, LW2]). In order to obtain this result, they introduced and studied a new kind of associative algebras, the so-called $\mathcal{Z}$-algebras (see also $[\mathrm{LP}$ ). (It turned out later on that these structures were closely related to the parafermion algebras that appeared in physics literature in the mid-eighties (cf. DU]).) Further applications of the $\mathcal{Z}$ algebra theory to the problem of constructing (what came to be called) fermionic representations may be found in e. g. [Ca2, $\mathrm{Ca} 3, \mathrm{MP} 1, \mathrm{P2}$, TX].

In this paper we make use of vertex operator techniques in order to determine certain sets of annihilating fields of standard $\tilde{\mathfrak{g}}[\sigma]$-modules for an affine Kac-Moody Lie algebra $\tilde{\mathfrak{g}}[\sigma]$ of arbitrary type. It turns out that these sets have a structure of loop $\tilde{\mathfrak{g}}[\sigma]$-modules of level 0 (see $[\mathrm{CP}$ for the definition). Moreover, these sets are minimal in the sense that they are irreducible loop $\tilde{\mathfrak{g}}[\sigma]$-modules (Theorems 2.9 and 2.1). We also get a description of the maximal submodule of the Verma $\tilde{\mathfrak{g}}[\sigma]$ module $M(\Lambda), \Lambda \in P_{+}$, in terms of these loop $\tilde{\mathfrak{g}}[\sigma]$-modules (Theorem 2.13). As a consequence, we obtain a characterization of standard $\tilde{\mathfrak{g}}[\sigma]$-modules by means of irreducible loop $\tilde{\mathfrak{g}}[\sigma]$-modules (Theorem 2.14). Partial analogues of this result have

1991 Mathematics Subject Classification. Primary: 17B67, 17B69; Secondary: 81R10.

Key words and phrases. Affine Lie algebras, standard modules, loop modules, vertex operator algebras, twisted modules, annihilating fields, combinatorial identities. 
proven to be very useful for combinatorial constructions of bases for standard modules in several particular cases (cf., e. g., Ca1, MP2, MP3]). The main ingredients used below are a combination between the untwisted representation theory of affine VOAs ( DL, Li1, MP2]) and Kac's classification of finite-order automorphisms of finite-dimensional simple Lie algebras ([K]), together with Li's results on deformations of vertex operator maps by means of certain endomorphisms associated with inner automorphisms ([Li2]).

The results presented here generalize those obtained in the untwisted case in MP2, §5] and [P1], and they may be of interest in their own right. Moreover, they may also provide an appropriate setting for the study of standard modules for affine Lie algebras of arbitrary type. Indeed, the above-mentioned results led in the untwisted case to the construction of bases for standard modules of arbitrary level for the rank two untwisted affine Lie algebra $A_{1}^{(1)}$ in MP2 (bases for these same modules had been previously constructed in [LP]). As a consequence, a series of combinatorial identities of Rogers-Ramanujan type was obtained (see also IMP3 for further applications). The representation theories of the rank two affine Lie algebras $A_{1}^{(1)}$ and $A_{2}^{(2)}$ are to a certain extent prototypical for the representation theory of all the untwisted respectively twisted affine Lie algebras. It seems therefore natural to investigate whether, for instance, standard $A_{2}^{(2)}$-modules of arbitrary level could be dealt with in a similar fashion by using the setting developed here. If successful, this approach may lead in particular to a combinatorial explanation of the dualitylike property for rank two affine Lie algebras discussed in [Bo] and is currently under study.

It is worth mentioning that a qualitative version of the results in [MP2, §5] was recently obtained in [FM] for the class of admissible representations (cf. [KW]) of untwisted affine Lie algebras. We believe that our results can be extended to admissible representations of affine Lie algebras of arbitrary type, which may then lead to further applications (like those in e. g. Ad).

Acknowledgements. I would like to thank Mirko Primc and Arne Meurman for numerous stimulating discussions, and the anonymous referee for his careful reading of this paper.

\section{Preliminaries And notations}

1.1. Realizations of affine Lie algebras. Let $\mathfrak{g}$ be a finite-dimensional simple complex Lie algebra. Fix a $C S A \mathfrak{t}$ of $\mathfrak{g}$ and let $\mu$ be an automorphism of $\mathfrak{g}$ of order $r(=1,2$, or 3$)$ induced by an automorphism of order $r$ of the Dynkin diagram of $\mathfrak{g}$ with respect to $\mathfrak{t}$. Let $\varepsilon$ be a primitive $r$-th root of unity and denote by $\mathfrak{g}_{[i]}$ the $\varepsilon^{i}$-eigenspace of $\mu$ in $\mathfrak{g}, i \in \mathbf{Z}_{r}$. Then $\mathfrak{g}_{[0]}$ is a simple subalgebra of $\mathfrak{g}$, the space $\mathfrak{t}_{[0]}:=\mathfrak{g}_{[0]} \cap \mathfrak{t}$ is a $C S A$ of $\mathfrak{g}_{[0]}$, and the $\mathfrak{g}_{[0]}$-modules $\mathfrak{g}_{[1]}$ and $\mathfrak{g}_{[-1]}$ are irreducible and contragredient. Set $l=\operatorname{rank} \mathfrak{g}_{[0]}$, and let $\left\{\beta_{1}, \ldots, \beta_{l}\right\} \subset \mathfrak{t}_{[0]}^{*}$ be a basis of the root system of $\mathfrak{g}_{[0]}$ and $\left\{E_{j}, F_{j}, H_{j} \mid j \in\{1, \ldots, l\}\right\}$ a corresponding set of canonical generators of $\mathfrak{g}_{[0]}$. Let $\beta_{0} \in \mathfrak{t}_{[0]}^{*}$ be the lowest weight of the $\mathfrak{g}_{[0]}$-module $\mathfrak{g}_{[1]}$, and let $E_{0}$ and $F_{0}$ be a lowest weight vector of the $\mathfrak{g}_{[0]}$-module $\mathfrak{g}_{[1]}$ respectively a highest weight vector of the $\mathfrak{g}_{[0]}$-module $\mathfrak{g}_{[-1]}$. We assume that $E_{0}$ and $F_{0}$ are normalized so that $\left[H_{0}, E_{0}\right]=2 E_{0}$, where $H_{0}=\left[E_{0}, F_{0}\right]$. For $i, j \in\{0,1, \ldots, l\}$ define $a_{i j}=\beta_{j}\left(H_{i}\right)$ and let $A=\left(a_{i j}\right)_{i, j=0}^{l}$. Then $A$ is a GCM of affine type, and so there are positive integers $a_{0}, \ldots, a_{l}$ such that $\left(a_{0}, \ldots, a_{l}\right) A^{t}=0$. Equivalently, 
there exist positive integers $\check{a_{0}}, \ldots, \check{a l}$ such that $\left(\check{a_{0}}, \ldots, \check{a_{l}}\right) A=0$. Both these sets of integers are assumed to be normalized so that $\operatorname{gcd}\left(a_{0}, \ldots, a_{l}\right)=\operatorname{gcd}\left(\check{a_{0}}, \ldots, \check{a_{l}}\right)$ $=1$. Then $h:=\sum_{j=0}^{l} a_{j}$ and $\check{h}:=\sum_{j=0}^{l} \check{a_{j}}$ are the Coxeter number respectively the dual Coxeter number of the matrix $A$, which will be denoted by $X_{N}^{(r)}$ if $\mathfrak{g}$ is of type $X_{N}(X=A, B, \ldots, G$ and $N \geq 1)$. We shall use the Dynkin diagrams of the affine GCMs as listed in KKLW, that is, with the vertex corresponding to the 0 -th index always occurring at the left-end of the diagram.

Let $\mathbf{s}=\left(s_{0}, s_{1}, \ldots, s_{l}\right)$ be a sequence of nonnegative relatively prime integers, and set $T=r \sum_{j=0}^{l} s_{j} a_{j}$. If $\eta$ is a primitive $T$-th root of unity, the conditions $\nu\left(H_{j}\right)=H_{j}, \nu\left(E_{j}\right)=\eta^{s_{j}} E_{j}, 0 \leq j \leq l$, define a $T$-th order automorphism $\nu$ of $\mathfrak{g}$, the so-called $\mathbf{s}$-automorphism. Since $\mathbf{C}$ is algebraically closed, every finite-order automorphism of $\mathfrak{g}$ arises in this way up to conjugation by an automorphism of $\mathfrak{g}$ ( $[\mathbb{K}$. Theorem 8.6]). Notice that in this terminology, the original diagram automorphism $\mu$ becomes the $(1,0, \ldots, 0)$-automorphism of $\mathfrak{g}$. Denote by $\mathfrak{g}_{(j)}$ the $\eta^{j}$-eigenspace of $\nu$ in $\mathfrak{g}$ for $j \in \mathbf{Z}_{T}$. The $\mathbf{Z}_{T}$-gradation $\mathfrak{g}=\coprod_{j \in \mathbf{Z}_{T}} \mathfrak{g}_{(j)}$ is accordingly called the $\nu$-gradation (or s-gradation) and a graded subspace of $\mathfrak{g}$ is said to be $\nu$-graded. Let $\langle\cdot, \cdot\rangle$ be a nondegenerate symmetric $\mathfrak{g}$-invariant bilinear form on $\mathfrak{g}$. Being a multiple of the Killing form, $\langle\cdot, \cdot\rangle$ is also $\nu$-invariant and remains nonsingular on the $C S A \mathfrak{t}_{[0]}$ of $\mathfrak{g}_{[0]}$. We may therefore identify $\mathfrak{t}_{[0]}$ with $\mathfrak{t}_{[0]}^{*}$ by means of the restricted form. Furthermore, we may assume that $\langle\cdot, \cdot\rangle$ is normalized so that $\left\langle\beta_{0}, \beta_{0}\right\rangle=2 \check{a_{0}} / r$, which then implies that $\check{a_{j}}=r\left\langle\beta_{j}, \beta_{j}\right\rangle a_{j} / 2$ for $j=0,1, \ldots, l$ (cf. KKLW, Proposition 1.1]). This normalization of the form $\langle\cdot, \cdot\rangle$ amounts to the condition that $\langle\alpha, \alpha\rangle=2$ whenever $\alpha \in \mathfrak{t}^{*}$ is a long root of $\mathfrak{g}$, in which case the Killing form equals $2 h\langle\cdot, \cdot\rangle$. Define the Lie algebras

$$
\hat{\mathfrak{g}}[\nu]=\oplus_{j=0}^{T-1} \mathfrak{g}_{(j)} \otimes t^{\frac{j}{T}} \mathbf{C}\left[t, t^{-1}\right] \oplus \mathbf{C} c, \quad \tilde{\mathfrak{g}}[\nu]=\hat{\mathfrak{g}}[\nu] \rtimes \mathbf{C} d
$$

by the conditions

$$
\begin{aligned}
& c \text { central, } c \neq 0,\left[d, a \otimes t^{m}\right]=m a \otimes t^{m}, \\
& {\left[a \otimes t^{m}, b \otimes t^{n}\right]=[a, b] \otimes t^{m+n}+m \delta_{m+n, 0}\langle a, b\rangle c,}
\end{aligned}
$$

for $m, n \in \frac{1}{T} \mathbf{Z}, a \in \mathfrak{g}_{(m T \bmod T)}, b \in \mathfrak{g}_{(n T \bmod T)}$. For $a \in \mathfrak{g}_{(j)}, n \in \mathbf{Z}$, we shall frequently use $a(n+j / T)$ and $\mathfrak{g}(n+j / T)$ to denote $a \otimes t^{n+j / T}$ and $\mathfrak{g}_{(j)} \otimes t^{n+j / T}$ respectively, and we often identify $\mathfrak{g}_{(0)}(0)$ with $\mathfrak{g}_{(0)}$. The space $\mathfrak{h}:=\mathfrak{t}_{[0]} \oplus \mathbf{C} c$ (respectively $\mathfrak{h}^{e}:=\mathfrak{h} \rtimes \mathbf{C} d$ ) is a $C S A$ of $\hat{\mathfrak{g}}[\nu]$ (respectively $\tilde{\mathfrak{g}}[\nu]$ ). Let $\delta \in \mathfrak{h} \mathfrak{e}^{*}$ be such that $\left.\delta\right|_{\mathfrak{h}}=0, \delta(d)=1$, and define $\alpha_{j} \in \mathfrak{h}^{e^{*}}$ by $\left.\alpha_{j}\right|_{\mathfrak{t}_{[0]}}=\beta_{j}, \alpha_{j}(c)=0, \alpha_{j}(d)=s_{j} T^{-1}$ if $j=1, \ldots, l$, and $\alpha_{0}=r^{-1} \delta-\sum_{j=1}^{l} a_{j} \alpha_{j}$, so that in particular $\alpha_{0}(d)=s_{0} T^{-1}$. For $j \in\{0,1, \ldots, l\}$ define also

$$
e_{j}=E_{j} \otimes t^{\frac{s_{j}}{T}}, \quad f_{j}=F_{j} \otimes t^{-\frac{s_{j}}{T}}, \quad h_{j}=H_{j}+\frac{2 s_{j}}{T\left\langle\beta_{j}, \beta_{j}\right\rangle} c .
$$

Then $\left\{e_{j}, f_{j}, h_{j}, d \mid j \in\{0,1, \ldots, l\}\right\}$ is a system of canonical generators of $\tilde{\mathfrak{g}}[\nu]$, viewed as the $\nu$-twisted affine Kac-Moody Lie algebra of rank $l+1$ associated to the GCM $A$, in what is called the s-realization of this algebra. Note that the canonical central element $c$ equals $\sum_{j=0}^{l} \check{a_{j}} h_{j}$, and let $\tilde{\mathfrak{g}}[\nu]_{i}:=\{a \in \tilde{\mathfrak{g}}[\nu] \mid[d, a]=i a\}, i \in \frac{1}{T} \mathbf{Z}$. The corresponding $\frac{1}{T} \mathbf{Z}$-gradation $\tilde{\mathfrak{g}}[\nu]=\coprod_{i \in \frac{1}{T} \mathbf{Z}} \tilde{\mathfrak{g}}[\nu]_{i}$ is then called the $\nu$-gradation of $\tilde{\mathfrak{g}}[\nu]$. Let as usual $\mathfrak{n}_{+}$and $\mathfrak{n}_{-}$denote the subalgebras of $\hat{\mathfrak{g}}[\nu]$ generated by $e_{0}, \ldots, e_{l}$ 
and by $f_{0}, \ldots, f_{l}$ respectively, so that one has the triangular decompositions

$$
\hat{\mathfrak{g}}[\nu]=\mathfrak{n}_{-} \oplus \mathfrak{h} \oplus \mathfrak{n}_{+}, \quad \tilde{\mathfrak{g}}[\nu]=\mathfrak{n}_{-} \oplus \mathfrak{h}^{e} \oplus \mathfrak{n}_{+},
$$

and corresponding decompositions of the universal enveloping algebras of $\hat{\mathfrak{g}}[\nu]$ and $\tilde{\mathfrak{g}}[\nu]$.

A $\hat{\mathfrak{g}}[\nu]$-module $V$ is said to be restricted and of level $l$ if $\mathfrak{g}_{(j)}(n+j / T) \cdot v=0$ for any $v \in V$ and $n \gg 0$ and $c$ acts as $l_{i d_{V}}$ on $V$. In particular, any highestweight module is restricted. Given a restricted $\hat{\mathfrak{g}}[\nu]$-module $V$ and $a \in \mathfrak{g}_{(j)}$, we shall consider the generating function of operators on $V$

$$
a(\nu ; z)=\sum_{n \in \mathbf{Z}} a(n+j / T) z^{-n-\frac{j}{T}-1} \in(\text { End } V)\left[\left[z^{1 / T}, z^{-1 / T}\right]\right] .
$$

We shall sometimes write $a_{n+j / T}$ when we think of $a(n+j / T)$ as a coefficient of $a(\nu ; z)$, and $a\left(\operatorname{id}_{\mathfrak{g}} ; z\right)$ will be denoted simply by $a(z)$. Let $M(\Lambda)$ be the Verma $\tilde{\mathfrak{g}}[\nu]$ module with highest weight $\Lambda \in \mathfrak{h}^{e^{*}}$. Denote by $M^{1}(\Lambda)$ its unique maximal proper submodule and let $L(\Lambda)=M(\Lambda) / M^{1}(\Lambda)$. Recall that a highest-weight $\tilde{\mathfrak{g}}[\nu]$-module $V$ with highest weight $\Lambda$ is standard if there exists $m \geq 1$ such that $f_{i}^{m} \cdot v_{\Lambda}=0$, $i=0,1, \ldots, l$, where $v_{\Lambda} \in V$ is a highest weight vector, which then implies that $\Lambda \in P_{+}:=\left\{\Lambda \in \mathfrak{h}^{e^{*}} \mid \Lambda\left(h_{i}\right) \in \mathbf{Z}_{\geq 0}\right.$ for $\left.i=0, \ldots, l\right\}$. Conversely, if $\Lambda \in P_{+}$then $L(\Lambda)$ is a standard $\tilde{\mathfrak{g}}[\nu]$-module and by [⿶, Corollary 10.4] one has that

$$
M^{1}(\Lambda)=\sum_{i=0}^{l} U\left(\mathfrak{n}_{-}\right) f_{i}^{\Lambda\left(h_{i}\right)+1} \cdot v_{\Lambda}
$$

Let finally $\Lambda_{i} \in P_{+}, 0 \leq i \leq l$, denote the fundamental weights determined by $\Lambda_{i}\left(h_{j}\right)=\delta_{i j}, \Lambda_{i}(d)=0$.

1.2. VOAs and modules. We refer to B, FHL, FLM for the definition of a vertex (operator) algebra, and to DD, Li1, Li2 for the different notions of weak module for a VOA. The definition of a twisted VOA-module used below may be found in e. g. Li2.

Let $(V, Y, \mathbf{1}, \omega)$ be a VOA, and recall that $\mathrm{id}_{V}$ together with the component operators of the field $Y(\omega, z)=\sum_{n \in \mathbf{Z}} L(n) z^{-n-2}$ generate a representation of the Virasoro algebra on $V$. Let further $\sigma$ be an automorphism of order $T$ of $V$ and $V^{k}=\{a \in V \mid \sigma(a)=\exp (2 k \pi i / T) a\}, 0 \leq k \leq T-1$, so that $V=\oplus_{k=0}^{T-1} V^{k}$. If $\left(M, Y_{M}\right)$ is a $\sigma$-twisted $V$-module and $a \in V^{k}, b \in V$, then

$$
Y_{M}(L(-1) b, z)=\frac{d}{d z} Y_{M}(b, z),
$$

and one has the following consequences of the defining axioms (cf., e. g., [i2]):

$$
\begin{aligned}
& z^{\frac{k}{T}} Y_{M}(a, z) \in(\text { End } M)\left[\left[z, z^{-1}\right]\right] \\
& {\left[Y_{M}\left(a, z_{1}\right), Y_{M}\left(b, z_{2}\right)\right]=\sum_{j=0}^{\infty} \frac{1}{j !}\left(\left(\frac{\partial}{\partial z_{2}}\right)^{j} z_{1}^{-1} \delta\left(\frac{z_{2}}{z_{1}}\right)\left(\frac{z_{2}}{z_{1}}\right)^{\frac{k}{T}}\right) Y_{M}\left(a_{j} b, z_{2}\right), } \\
& Y_{M}\left(Y\left(a, z_{0}\right) b, z_{2}\right)=\operatorname{Res}_{z_{1}}\left(\frac{z_{1}-z_{0}}{z_{2}}\right)^{\frac{k}{T}}\left[\begin{array}{l}
z_{0}^{-1} \delta\left(\frac{z_{1}-z_{2}}{z_{0}}\right) Y_{M}\left(a, z_{1}\right) Y_{M}\left(b, z_{2}\right) \\
\left.-z_{0}^{-1} \delta\left(\frac{-z_{2}+z_{1}}{z_{0}}\right) Y_{M}\left(b, z_{2}\right) Y_{M}\left(a, z_{1}\right)\right] .
\end{array}\right.
\end{aligned}
$$


We now describe briefly the so-called affine VOAs. Let $\mathfrak{g}$ be a finite-dimensional simple Lie algebra with the form $\langle\cdot, \cdot\rangle$ normalized as in $\S 1.1$, and form the untwisted affine Kac-Moody algebra $\tilde{\mathfrak{g}}=\hat{\mathfrak{g}} \rtimes \mathbf{C} d$ as in (1.1)-(1.2). Set $\tilde{\mathfrak{g}}_{\geq 0}=\oplus_{n \geq 0} \mathfrak{g}(n) \oplus \mathbf{C} c \oplus$ $\mathbf{C} d$ and let $-\hbar \neq l \in \mathbf{C}$. Recall from $\S 1.1$ the fundamental weight $\Lambda_{0} \in P_{+}$and define a $\tilde{\mathfrak{g}}_{\geq 0}$-module structure on $\mathbf{C}$ by $c \cdot 1=l, d \cdot 1=0, \mathfrak{g}(n) \cdot 1=0$ for $n \geq 0$. We may then form the Weyl module (or generalized Verma module) $N\left(l \Lambda_{0}\right)=$ $U(\tilde{\mathfrak{g}}) \otimes_{U(\tilde{\mathfrak{g}}>0)} \mathbf{C}$, which is the so-called vacuum representation of level $l$ of $\tilde{\mathfrak{g}}$. Note that $N\left(l \Lambda_{0}\right)$ is a restricted $\tilde{\mathfrak{g}}$-module such that $N\left(l \Lambda_{0}\right) \cong U\left(\oplus_{n<0} \mathfrak{g}(n)\right)$ as vector spaces, and that we may identify $\mathfrak{g}(-1) \otimes 1$ with $\mathfrak{g}$. Set $\mathbf{1}=1 \otimes 1 \in N\left(l \Lambda_{0}\right)$ and define the element

$$
\omega=\frac{1}{2\left(l+h^{j}\right)} \sum_{j=1}^{\operatorname{dim} \mathfrak{g}} a^{j}(-1)^{2} \mathbf{1} \in N\left(l \Lambda_{0}\right),
$$

where $\left\{a^{j} \mid j \in\{1, \ldots, \operatorname{dim} \mathfrak{g}\}\right\}$ is an orthonormal basis of $\mathfrak{g}$ with respect to $\langle\cdot, \cdot\rangle$. Recall from (1.5) the series $a(z)$ in this case and define the map

$$
\begin{gathered}
Y: \mathfrak{g}(-1) \otimes 1 \longrightarrow\left(\text { End } N\left(l \Lambda_{0}\right)\right)\left[\left[z, z^{-1}\right]\right] \\
Y(a(-1) \otimes 1, z)=a(z), a \in \mathfrak{g} .
\end{gathered}
$$

One can show (cf., e. g., MP2, Theorem 2.6]) that $Y$ extends uniquely to $N\left(l \Lambda_{0}\right)$ in such a way that $N\left(l \Lambda_{0}\right)$ becomes a VOA with vacuum vector $\mathbf{1}$ and Virasoro element $\omega$ such that $\mathfrak{g}(-1) \otimes 1=N\left(l \Lambda_{0}\right)_{1}$ (the weight one subspace of $N\left(l \Lambda_{0}\right)$ ). Moreover, given any restricted $\hat{\mathfrak{g}}$-module $M$ of level $l$, there is a canonical extension to $N\left(l \Lambda_{0}\right)$ of the map

$$
\begin{gathered}
Y_{M}: \mathfrak{g}(-1) \otimes 1 \longrightarrow(\text { End } M)\left[\left[z, z^{-1}\right]\right] \\
Y_{M}(a(-1) \otimes 1, z)=a(z), a \in \mathfrak{g},
\end{gathered}
$$

such that $\left(M, Y_{M}\right)$ becomes a weak $N\left(l \Lambda_{0}\right)$-module (DD, Li1, MP2]). Let finally $N^{1}\left(l \Lambda_{0}\right)$ be the unique maximal proper $\tilde{\mathfrak{g}}$-submodule of $N\left(l \Lambda_{0}\right)$ and notice that we may identify the irreducible quotient $N\left(l \Lambda_{0}\right) / N^{1}\left(l \Lambda_{0}\right)$ with the $\tilde{\mathfrak{g}}$-module $L\left(l \Lambda_{0}\right)$ defined in $\S 1.1$. We summarize some of the results of the above-mentioned papers in

Theorem 1.1. For each $l \neq-h,\left(N\left(l \Lambda_{0}\right), Y, \mathbf{1}, \omega\right)$ is a VOA of rank $\frac{l \operatorname{dimg}}{l+h}$ and any restricted $\hat{\mathfrak{g}}$-module of level $l$ is a weak $N\left(l \Lambda_{0}\right)$-module. Every $\tilde{\mathfrak{g}}$-submodule of $N\left(l \Lambda_{0}\right)$ is an ideal of $N\left(l \Lambda_{0}\right)$ viewed as a VOA. In particular, there exists an induced structure of simple VOA on $L\left(l \Lambda_{0}\right)$.

Any automorphism $\sigma$ of order $T$ of $\mathfrak{g}$ preserves the form $\langle\cdot, \cdot\rangle$ and induces a Lie algebra automorphism of $\tilde{\mathfrak{g}}$. It follows from the associator formula for VOAs that $\sigma$ also induces VOA automorphisms of $N\left(l \Lambda_{0}\right)$ and $L\left(l \Lambda_{0}\right)$ respectively, and we denote these induced automorphisms again by $\sigma$. If $M$ is a restricted $\hat{\mathfrak{g}}[\sigma]$-module of level $l$, the map

$$
\begin{gathered}
Y_{M}^{\sigma}: \mathfrak{g}(-1) \otimes 1 \longrightarrow(\text { End } M)\left[\left[z^{1 / T}, z^{-1 / T}\right]\right] \\
Y_{M}^{\sigma}(a(-1) \otimes 1, z)=a(\sigma ; z),
\end{gathered}
$$

for $a \in \mathfrak{g}_{(j)}, j=0, \ldots, T-1$, has a unique extension to $N\left(l \Lambda_{0}\right)$ that makes $\left(M, Y_{M}^{\sigma}\right)$ a weak $\sigma$-twisted $N\left(l \Lambda_{0}\right)$-module. This is a consequence of the theory of local systems of twisted vertex operators developed in [Li2], where the following $\sigma$-twisted counterpart of Theorem 1.1 was obtained: 
Theorem 1.2. Let $l \neq-\hbar$ be a complex number. Then any restricted $\hat{\mathfrak{g}}[\sigma]$-module of level $l$ is a weak $\sigma$-twisted $N\left(l \Lambda_{0}\right)$-module.

\section{MAin RESUlts}

We use the setting of Section 1 throughout: $\mathfrak{g}$ is a finite-dimensional simple Lie algebra with the form $\langle\cdot, \cdot\rangle$ normalized as in $\S 1.1, \sigma$ is an automorphism of order $T$ of $\mathfrak{g}, \mathfrak{g}=\coprod_{j \in \mathbf{Z}_{T}} \mathfrak{g}_{(j)}$ denotes the $\sigma$-gradation of $\mathfrak{g}$, and $M$ is a restricted $\tilde{\mathfrak{g}}[\sigma]$-module of level $k \in \mathbf{C}$ (in particular, $M$ could be a Verma module). Recall from Theorem 1.2 that $\left(M, Y_{M}^{\sigma}\right)$ is a weak $\sigma$-twisted $N\left(k \Lambda_{0}\right)$-module and let $R$ be a $\sigma$-invariant subspace of $N\left(k \Lambda_{0}\right)$ with $\sigma$-decomposition $R=\coprod_{j \in \mathbf{Z}_{T}} R^{j}$. We also assume that $R$ is invariant under both $\mathfrak{g}(0)$ and $L(0)\left(=\operatorname{Res}_{z} z Y(\omega, z)\right)$. Define the space

$$
\bar{R}_{\sigma}=\mathbf{C}-\operatorname{span}\left\{r_{n} \mid r \in R, n \in \frac{1}{T} \mathbf{Z}\right\} \subset \text { End } M,
$$

where $Y_{M}^{\sigma}(r, z)=\sum_{n \in \frac{1}{T}} \mathbf{z}_{n} r z^{-n-1}$, and let $\eta=\exp (2 \pi i / T)$. We denote by $\sigma$ as well the linear automorphism of (End $M)\left[\left[z^{1 / T}, z^{-1 / T}\right]\right]$ determined by $\sigma f\left(z^{1 / T}\right)=$ $f\left(\eta^{-1} z^{1 / T}\right)$. It follows from (1.8) that $\sigma\left(v_{n}\right)=(\sigma(v))_{n}$ for $v \in N\left(k \Lambda_{0}\right), n \in \frac{1}{T} \mathbf{Z}$, so that in particular $\bar{R}_{\sigma}$ is $\sigma$-stable and thus

$$
\bar{R}_{\sigma}=\coprod_{j \in \mathbf{Z}_{T}} \bar{R}_{\sigma}^{j}
$$

where $\bar{R}_{\sigma}^{j}$ is the $\eta^{j}$-eigenspace of $\sigma$ in $\bar{R}_{\sigma}$. Furthermore, the twisted commutator formula (1.9) together with the derivation property (1.7) yield

$$
\left[L(0), Y_{M}^{\sigma}(v, z)\right]=Y_{M}^{\sigma}(L(0) v, z)+z \frac{d}{d z} Y_{M}^{\sigma}(v, z)
$$

for $v \in N\left(k \Lambda_{0}\right)$, with $L(0)=\operatorname{Res}_{z} z Y_{M}^{\sigma}(\omega, z)$ in the left-hand side. Note that we may identify $\tilde{\mathfrak{g}}[\sigma]$ with $\hat{\mathfrak{g}}[\sigma] \rtimes \mathbf{C} L(0) \subset$ End $M$. Since $R$ is $L(0)$-invariant, (2.3) implies that $L(0)$ induces a $\frac{1}{T} \mathbf{Z}$-gradation on $\bar{R}_{\sigma}$ :

$$
\bar{R}_{\sigma}=\coprod_{n \in \frac{1}{T} \mathbf{z}} \bar{R}_{\sigma}(n)
$$

where $\bar{R}_{\sigma}(n)=\left\{p \in \bar{R}_{\sigma} \mid[L(0), p]=n p\right\}$ for $n \in \frac{1}{T} \mathbf{Z}$. The gradations (2.2) and (2.4) are compatible in the sense that

$$
\bar{R}_{\sigma}^{j}=\coprod_{n \in \frac{1}{T} \mathbf{Z}, n T \bmod T \equiv j} \bar{R}_{\sigma}(n) \text { for } j \in \mathbf{Z}_{T} \text { and } \bar{R}_{\sigma}(n)=\coprod_{j \in \mathbf{Z}_{T}} \bar{R}_{\sigma}(n)^{j} \text { for } n \in \frac{1}{T} \mathbf{Z},
$$

where $\bar{R}_{\sigma}(n)^{j}=\bar{R}_{\sigma}^{j} \cap \bar{R}_{\sigma}(n)$. Note that (1.8) implies that $\bar{R}_{\sigma}(n)$ is in fact $\sigma$ homogeneous, since $\bar{R}_{\sigma}(n)^{j}=0$ unless $j \equiv n T \bmod T$.

Theorem 2.1. (i) Let $R$ and $M$ be as above and assume that $\mathfrak{g}(n) R=0$ for $n \in$ $\mathbf{Z}_{>0}$. Then

$$
\left[x(m), r_{n}\right]=(x(0) r)_{m+n}
$$

for all $x \in \mathfrak{g}, r \in R$, and $m, n \in \frac{1}{T} \mathbf{Z}$, so that $\bar{R}_{\sigma}$ becomes a loop module under the adjoint action of $\tilde{\mathfrak{g}}[\sigma]$. Conversely, if $M$ is a faithful weak $\sigma$-twisted $N\left(k \Lambda_{0}\right)$-module and (2.6) holds, then

$$
\mathfrak{g}(n) R=0 \quad \forall n \in \mathbf{Z}_{>0} .
$$


(ii) $\bar{R}_{\sigma}$ is an irreducible loop $\tilde{\mathfrak{g}}[\sigma]$-module if $R$ is a nontrivial irreducible $\mathfrak{g}$-module. Moreover, if $M$ is a faithful weak $\sigma$-twisted $N\left(k \Lambda_{0}\right)$-module, then the converse is also true.

Proof. (i) Suppose that $\mathfrak{g}(n) R=0$ for all positive integers $n$. It suffices to prove (2.6) for $\sigma$-homogeneous elements $x \in \mathfrak{g}_{(j)}, r \in R^{k}$, where $j, k \in\{0,1, \ldots, T-1\}$. Note first that $x(0) r \in R^{j+k}$ and let $m \in \frac{j}{T}+\mathbf{Z}, n \in \frac{k}{T}+\mathbf{Z}$ be fixed. Then (1.8) and (1.9) imply that

$$
\left[x\left(\sigma ; z_{1}\right), Y_{M}^{\sigma}\left(r, z_{2}\right)\right]=\sum_{p \in \mathbf{Z}, q \in \frac{j+k}{T}+\mathbf{Z}}(x(0) r)_{q} z_{1}^{-p-\frac{j}{T}-1} z_{2}^{-q+p+\frac{j}{T}-1} .
$$

On the other hand

$$
\left[x\left(\sigma ; z_{1}\right), Y_{M}^{\sigma}\left(r, z_{2}\right)\right]=\sum_{s \in \frac{j}{T}+\mathbf{Z}, t \in \frac{k}{T}+\mathbf{Z}}\left[x(s), r_{t}\right] z_{1}^{-s-1} z_{2}^{-t-1}
$$

by (1.8), and then (2.6) follows by comparing the coefficients of $z_{1}^{-m-1} z_{2}^{-n-1}$ in the right-hand sides of (2.8) and (2.9) respectively.

Assume now that $M$ is a faithful weak $\sigma$-twisted $N\left(k \Lambda_{0}\right)$-module and that (2.6) holds, and let $N \in \mathbf{Z}_{\geq 0}$ be such that $x(p) r=0$ for all $p \geq N+1$. Then (2.6) and (2.9) yield

$$
\left[x\left(\sigma ; z_{1}\right), Y_{M}^{\sigma}\left(r, z_{2}\right)\right]=z_{1}^{-1} \delta\left(\frac{z_{2}}{z_{1}}\right)\left(\frac{z_{2}}{z_{1}}\right)^{\frac{j}{T}} Y_{M}^{\sigma}\left(x(0) r, z_{2}\right)
$$

Thus

$$
\sum_{i=1}^{N} \frac{1}{i !}\left(\left(\frac{\partial}{\partial z_{2}}\right)^{i} z_{1}^{-1} \delta\left(\frac{z_{2}}{z_{1}}\right)\left(\frac{z_{2}}{z_{1}}\right)^{\frac{j}{T}}\right) Y_{M}^{\sigma}\left(x(i) r, z_{2}\right)=0
$$

by (1.9). Using [Li2, Lemma 2.3] one gets that $Y_{M}^{\sigma}\left(x(i) r, z_{2}\right)=0$ for $1 \leq i \leq N$. Therefore $x(i) r=0$ for $i \in\{1, \ldots, N\}$ as well (since $M$ is faithful), which proves (2.7).

(ii) We first prove the second statement. Suppose that $\bar{R}_{\sigma}$ is irreducible. If $R$ were a 1-dimensional trivial $\mathfrak{g}$-module, then $\mathbf{C} r_{n}$ would be a nonzero $\tilde{\mathfrak{g}}[\sigma]$-submodule of $\bar{R}_{\sigma}$ for each nonzero $r \in R$ and $n \in \frac{1}{T} \mathbf{Z}$ satisfying $r_{n} \neq 0$ (such elements exist since $M$ is faithful), hence a contradiction. Suppose now that $S \subset R$ is a nonzero proper $\mathfrak{g}$-submodule of $R$. Then there exists $0 \neq r \in R_{d} \backslash S_{d}$ for some $d \in \mathbf{Z}$, where $R=\coprod_{n \in \mathbf{Z}} R_{n}$ and $S=\coprod_{n \in \mathbf{Z}} S_{n}$ are the $L(0)$-gradations of $R$ and $S$ respectively. Note that $\left(\overline{S_{d}}\right)_{\sigma} \subset\left(\overline{R_{d}}\right)_{\sigma}$ are $\tilde{\mathfrak{g}}[\sigma]$-submodules of $\bar{R}_{\sigma}$ by $(2.6)$, and thus

$$
\left(\overline{S_{d}}\right)_{\sigma}=\left(\overline{R_{d}}\right)_{\sigma}=\bar{R}_{\sigma}
$$

since $\bar{R}_{\sigma}$ is irreducible. It follows from (2.3), (2.4) and (2.10) that there exist $m \in \frac{1}{T} \mathbf{Z}$ and $s \in S_{d}$ such that $r_{m} \neq 0$ and $r_{m}=s_{m}$. Then (2.6) implies that $x(0) t=0$ for all $x \in \mathfrak{g}$, where $t:=r-s$. Consequently, $\bar{T}_{\sigma}:=\mathbf{C}$-span $\left\{t_{l} \mid l \in \frac{1}{T} \mathbf{Z}\right\}$ is a (trivial) $\tilde{\mathfrak{g}}[\sigma]$-submodule of $\bar{R}_{\sigma}$ by (2.6). Clearly, $r_{m} \notin \bar{T}_{\sigma}$, so that $\bar{T}_{\sigma} \neq \bar{R}_{\sigma}$ and thus $\bar{T}_{\sigma}=0$. Hence $t=0$ (again by the faithfulness of $M$ ), and one gets that $r=s \in S_{d}$, which is a contradiction. $R$ must therefore be an irreducible $\mathfrak{g}$-module.

Conversely, suppose that $R$ is a nontrivial irreducible $\mathfrak{g}$-module. Then $R$ is $L(0)$-homogeneous of some conformal weight $d \in \mathbf{Z}$. Recall (2.5) and notice that

$$
\bar{R}_{\sigma}(n)=\left\{r_{d-n-1} \mid r \in R\right\}, \quad n \in \frac{1}{T} \mathbf{Z} .
$$


Let $Z$ be a nonzero $\tilde{\mathfrak{g}}[\sigma]$-submodule of $\bar{R}_{\sigma}$. Then $Z=\coprod_{n \in \frac{1}{T} \mathbf{Z}} Z(n)(Z$ being $L(0)$ stable), where $Z(n)=\{z \in Z \mid[L(0), z]=n z\} \subset \bar{R}_{\sigma}(n)$. By (2.11) there exist $n \in \frac{1}{T} \mathbf{Z}$ and $r \in R$ such that $0 \neq r_{d-n-1} \in Z(n)$, and it follows from (2.6) that

$$
(x(0) r)_{d-m-1}=\left[x(n-m), r_{d-n-1}\right] \in Z(m)
$$

for every $x \in \mathfrak{g}$ and $m \in \frac{1}{T} \mathbf{Z}$. Since $R$ is irreducible, one has $R=U(\mathfrak{g}) \cdot r$, and then by iterating (2.12) one gets $\bar{R}_{\sigma}(m) \subset Z(m)$ for all $m \in \frac{1}{T} \mathbf{Z}$. Therefore $Z=\bar{R}_{\sigma}$ and the proof is complete.

Remark 2.2. It follows from the twisted associator formula (1.10) and the irreducibility of $L\left(l \Lambda_{0}\right)$ that level $l$ standard $\hat{\mathfrak{g}}[\sigma]$-modules are in fact faithful $\sigma$-twisted $L\left(l \Lambda_{0}\right)$-modules. It would be interesting to see whether the Verma $\tilde{\mathfrak{g}}[\sigma]$-module $M(\Lambda)$ is itself a faithful $\sigma$-twisted $N\left(k \Lambda_{0}\right)$-module, where $\Lambda \in P_{+}$is such that $\Lambda(c)=k \in \mathbf{Z}_{+}$. This question is obviously related to the classification of submodules of $N\left(k \Lambda_{0}\right)$, which in turn lies close to the problem of classifying annihilating ideals of standard (or even admissible) $\tilde{\mathfrak{g}}[\sigma]$-modules (cf. FM]).

In the remainder of this section, $k$ is assumed to be a fixed positive integer. The next result will be used in the proofs of Theorems 2.9 and 2.13 .

Proposition 2.3 ([Li2]). Let $V$ be a VOA, let $\sigma$ be an automorphism of order $S$ of $V$ and $h \in V$ such that $L(n) h=\delta_{n, 0} h, \sigma(h)=h,\left[h_{m}, h_{n}\right]=0$ for $m, n \in \mathbf{Z}_{\geq 0}$. Assume that $h(0)$ acts semisimply on $V$ and that $\operatorname{Spec}(h(0)) \subset \frac{1}{T} \mathbf{Z}$ for some $T \in$ $\mathbf{Z}_{>0}$, so that $\sigma_{h}:=\exp (2 \pi i h(0))$ is an automorphism of $V$ satisfying $\sigma_{h}^{T}=\mathrm{id}_{V}$. Let $\left(M, Y_{M}^{\sigma}\right)$ be a $\sigma$-twisted $V$-module and define

$$
\Delta(h, z)=z^{h(0)} \exp \left(\sum_{n=1}^{\infty}(-1)^{n-1} \frac{h(n)}{n} z^{-n}\right), \quad \bar{Y}_{M}^{\sigma}(a, z)=Y_{M}^{\sigma}(\Delta(h, z) a, z)
$$

for $a \in V$. Then $\left(M, \bar{Y}_{M}^{\sigma}(\cdot, z)\right)$ is a weak $\left(\sigma \sigma_{h}\right)$-twisted $V$-module.

Remark 2.4. As pointed out in [Li2], Proposition 2.3 gives an isomorphism between $\hat{\mathfrak{g}}[\sigma]$ and $\hat{\mathfrak{g}}\left[\sigma \sigma_{h}\right]$. Furthermore, $\left(M, \bar{Y}_{M}^{\sigma}\right)$ is irreducible if $\left(M, Y_{M}^{\sigma}\right)$ is irreducible $\left(\Delta(h, z)\right.$ being invertible), and any $\sigma_{h}$-twisted $V$-module can be constructed from a $V$-module.

The following well-known result will be needed for the proof of Theorem 2.13:

Proposition 2.5 (枭). Let $\mathfrak{g}$ be a simple finite-dimensional Lie algebra, let $\mathfrak{t}$ be $a$ CSA of $\mathfrak{g}$ and let $\Pi=\left\{\alpha_{1}, \ldots, \alpha_{n}\right\}$ be a set of simple roots. Let $\sigma \in \operatorname{Aut}(\mathfrak{g})$ be such that $\sigma^{T}=\mathrm{id}_{\mathfrak{g}}$. Then $\sigma$ is conjugate to an automorphism of $\mathfrak{g}$ of the form

$$
\mu \exp \left(\operatorname{ad}\left(\frac{2 \pi i}{T} h\right)\right), \quad h \in \mathfrak{t}_{[0]},
$$

where $\mu$ is a diagram automorphism preserving $\mathfrak{t}$ and $\Pi, \mathfrak{t}_{[0]}$ is the fixed-point set of $\mu$ in $\mathfrak{t}$, and $\alpha_{i}(h) \in \mathbf{Z}$ for $i \in\{1, \ldots, n\}$.

As in $\S 1.1$, we fix a $C S A$ of $\mathfrak{g}$ and denote it by $\mathfrak{t}$. Let $\Phi$ be the root system of $\mathfrak{g}$ and let $\Pi=\left\{\alpha_{1}, \ldots, \alpha_{n}\right\} \subset \mathfrak{t}^{*}$ be a basis of $\Phi$ enumerated as in $[\mathrm{K}$, Table Fin]. Choose root vectors $x_{\alpha} \in \mathfrak{g}_{\alpha}$ such that $h_{\alpha}:=\left[x_{\alpha}, x_{-\alpha}\right]$ satisfies $\alpha\left(h_{\alpha}\right)=2$ for $\alpha \in \Phi$, and let $\theta$ be the highest root. Notice that with the above normalizations one always has $\left\langle x_{\theta}, x_{-\theta}\right\rangle=1$. We shall use the following result from the untwisted representation theory (cf. Li1, MP2]): 
Proposition 2.6. Let $M$ be an integrable $\tilde{\mathfrak{g}}$-module of level $k$. Then $x_{\alpha}(z)^{t k+1}=0$ acting on $M$, where $t=1$ if $\alpha$ is a long root, $t=2$ if $\alpha$ is a short root and $\mathfrak{g}$ is not of type $G_{2}$, and $t=3$ if $\alpha$ is a short root and $\mathfrak{g}$ is of type $G_{2}$.

Let now $\mu$ be a diagram automorphism of $\mathfrak{g}$ induced by an automorphism $\bar{\mu}$ of the Dynkin diagram of $\mathfrak{g}$ with respect to $(\mathfrak{t}, \Pi)$. It is well-known that the subgroup of $\operatorname{Aut}(\mathfrak{g})$ generated by all such diagram automorphisms is isomorphic to the symmetric group $\mathcal{S}_{m}$, where $m=1$ for $B_{n}, C_{n}, E_{7}, E_{8}, F_{4}$ and $G_{2}, m=2$ for $A_{n}$ $(n \geq 2), D_{n}(n \geq 3, n \neq 4)$ and $E_{6}$, and $m=3$ for $D_{4}$. Let $r(=1,2$, or 3$)$ be the order of $\mu$, so that in particular one has the $\mu$-decompositions $\mathfrak{g}=\coprod_{j \in \mathbf{Z}_{r}} \mathfrak{g}_{[j]}$ and (since $\mathfrak{t}$ is obviously $\mu$-stable) $\mathfrak{t}=\coprod_{j \in \mathbf{Z}_{r}} \mathfrak{t}_{[j]}$. We shall concentrate on the five cases when $r \geq 2$, namely when $\mathfrak{g}$ is of type $A_{2 l}, A_{2 l-1}, D_{l+1}, E_{6}$ or $D_{4}$, and $r=2,2,2,2$ or 3 respectively (for $r=3$ there are two equivalent automorphisms of this type and we just choose one of them). Recall that $\mathfrak{g}_{[0]}$ is a simple subalgebra of $\mathfrak{g}$ such that $\mathfrak{t}_{[0]}=\mathfrak{g}_{[0]} \cap \mathfrak{t}$ is a $C S A$ of $\mathfrak{g}_{[0]}$. More specifically, $\mathfrak{g}_{[0]}$ is of type $B_{l}$, $A_{1}, C_{l}, B_{l}, F_{4}, G_{2}$ whenever $\mathfrak{g}$ is of type $A_{2 l}(l \geq 2), A_{2}, A_{2 l-1}, D_{l+1}, E_{6}, D_{4}$ respectively. Recall also the elements $E_{i}, F_{i}, H_{i} \in \mathfrak{g}$ and the simple roots $\beta_{i} \in \mathfrak{t}_{[0]}^{*}$, $i=0,1, \ldots l$, defined in $\S 1.1$. These are given explicitly in $[\mathbb{K}]$, where an additional element $\theta^{0} \in \Phi$ is introduced as follows (in case 1 we switched the indexes 0 and $l$ as compared with [K]):

Case 1: $\mathfrak{g}=A_{2 l}, r=2 ; \bar{\mu}\left(\alpha_{i}\right)=\alpha_{2 l-i+1}(1 \leq i \leq 2 l), \mu\left(h_{\alpha}\right)=h_{\bar{\mu}(\alpha)}$,

$$
\begin{aligned}
& \mu\left(x_{\alpha}\right)=(-1)^{1+h t(\alpha)} x_{\bar{\mu}(\alpha)} ; \theta=\sum_{i=1}^{2 l} \alpha_{i}=\theta^{0},-\beta_{0}=\left.\theta^{0}\right|_{\mathfrak{t}_{[0]}}, \\
& \beta_{i}=\left.\alpha_{i}\right|_{\mathfrak{t}_{[0]}}=\left.\alpha_{2 l-i+1}\right|_{\mathfrak{t}_{[0]}}(1 \leq i \leq l) ; E_{0}=x_{-\theta}, \\
& E_{i}=x_{\alpha_{i}}+x_{\alpha_{2 l-i+1}}(1 \leq i \leq l-1), E_{l}=\sqrt{2}\left(x_{\alpha_{l}}+x_{\alpha_{l+1}}\right) ; F_{0}=x_{\theta}, \\
& F_{i}=x_{-\alpha_{i}}+x_{-\alpha_{2 l-i+1}}(1 \leq i \leq l-1), F_{l}=\sqrt{2}\left(x_{-\alpha_{l}}+x_{-\alpha_{l+1}}\right) ; \\
& H_{i}=\left[E_{i}, F_{i}\right](0 \leq i \leq l) .
\end{aligned}
$$

Case 2: $\mathfrak{g}=A_{2 l-1}, r=2 ; \bar{\mu}\left(\alpha_{i}\right)=\alpha_{2 l-i}(1 \leq i \leq 2 l-1), \mu\left(h_{\alpha}\right)=h_{\bar{\mu}(\alpha)}$,

$$
\begin{aligned}
& \mu\left(x_{\alpha}\right)=x_{\bar{\mu}(\alpha)} ; \theta^{0}=\theta-\alpha_{2 l-1},-\beta_{0}=\left.\frac{1}{2}\left(\theta^{0}+\bar{\mu}\left(\theta^{0}\right)\right)\right|_{\mathfrak{t}_{[0]}}, \\
& \beta_{i}=\left.\alpha_{i}\right|_{\mathfrak{t}_{[0]}}=\left.\alpha_{2 l-i}\right|_{\mathfrak{t}_{[0]}}(1 \leq i \leq l) ; E_{0}=x_{-\theta^{0}}-x_{-\bar{\mu}\left(\theta^{0}\right)}, \\
& E_{i}=x_{\alpha_{i}}+x_{\alpha_{2 l-i}}(1 \leq i \leq l-1), E_{l}=x_{\alpha_{l}} ; F_{0}=x_{\theta^{0}}-x_{\bar{\mu}\left(\theta^{0}\right)}, \\
& F_{i}=x_{-\alpha_{i}}+x_{-\alpha_{2 l-i}}(1 \leq i \leq l-1), F_{l}=x_{-\alpha_{l}} ; H_{i}=\left[E_{i}, F_{i}\right](0 \leq i \leq l) .
\end{aligned}
$$

Case 3: $\mathfrak{g}=D_{l+1}, r=2 ; \bar{\mu}\left(\alpha_{i}\right)=\alpha_{i}(1 \leq i \leq l-1), \bar{\mu}\left(\alpha_{l}\right)=\alpha_{l+1}, \bar{\mu}\left(\alpha_{l+1}\right)=\alpha_{l}$,

$$
\begin{aligned}
& \mu\left(h_{\alpha}\right)=h_{\bar{\mu}(\alpha)}, \mu\left(x_{\alpha}\right)=x_{\bar{\mu}(\alpha)} ; \theta=\alpha_{1}+2 \sum_{i=2}^{l-1} \alpha_{i}+\alpha_{l}+\alpha_{l+1}, \\
& \theta^{0}=\frac{1}{2}\left(\theta+\alpha_{1}+\alpha_{l}-\alpha_{l+1}\right),-\beta_{0}=\left.\frac{1}{2}\left(\theta^{0}+\bar{\mu}\left(\theta^{0}\right)\right)\right|_{\mathfrak{t}_{[0]}}, \\
& \beta_{i}=\left.\alpha_{i}\right|_{\mathfrak{t}_{[0]}}(1 \leq i \leq l-1), \beta_{l}=\left.\alpha_{l}\right|_{\mathfrak{t}_{[0]}}=\left.\alpha_{l+1}\right|_{\mathfrak{t}_{[0]}} ; \\
& E_{0}=x_{-\theta^{0}}-x_{-\bar{\mu}\left(\theta^{0}\right)}, E_{i}=x_{\alpha_{i}}(1 \leq i \leq l-1), E_{l}=x_{\alpha_{l}}+x_{\alpha_{l+1}} ; \\
& F_{0}=x_{\theta^{0}}-x_{\bar{\mu}\left(\theta^{0}\right)}, F_{i}=x_{-\alpha_{i}}(1 \leq i \leq l-1), F_{l}=x_{-\alpha_{l}}+x_{-\alpha_{l+1}} ; \\
& H_{i}=\left[E_{i}, F_{i}\right](0 \leq i \leq l) .
\end{aligned}
$$


Case 4: $\mathfrak{g}=E_{6}, r=2 ; \bar{\mu}\left(\alpha_{1}\right)=\alpha_{5}, \bar{\mu}\left(\alpha_{2}\right)=\alpha_{4}, \bar{\mu}\left(\alpha_{3}\right)=\alpha_{3}, \bar{\mu}\left(\alpha_{6}\right)=\alpha_{6}$,

$\mu\left(h_{\alpha}\right)=h_{\bar{\mu}(\alpha)}, \mu\left(x_{\alpha}\right)=x_{\bar{\mu}(\alpha)} ; \theta=\alpha_{1}+2 \alpha_{2}+3 \alpha_{3}+2 \alpha_{4}+\alpha_{5}+2 \alpha_{6}$,

$\theta^{0}=\theta-\alpha_{3}-\alpha_{4}-\alpha_{6},-\beta_{0}=\left.\frac{1}{2}\left(\theta^{0}+\bar{\mu}\left(\theta^{0}\right)\right)\right|_{\mathfrak{t}_{[0]}}, \beta_{1}=\left.\alpha_{1}\right|_{\mathfrak{t}_{[0]}}=\left.\alpha_{5}\right|_{\mathfrak{t}_{[0]}}$,

$\beta_{2}=\left.\alpha_{2}\right|_{\mathfrak{t}_{[0]}}=\left.\alpha_{4}\right|_{\mathfrak{t}_{[0]}}, \beta_{3}=\left.\alpha_{3}\right|_{\mathfrak{t}_{[0]}}, \beta_{4}=\left.\alpha_{6}\right|_{\mathfrak{t}_{[0]}} ; E_{0}=x_{-\theta^{0}}-x_{-\bar{\mu}\left(\theta^{0}\right)}$,

$E_{1}=x_{\alpha_{1}}+x_{\alpha_{5}}, E_{2}=x_{\alpha_{2}}+x_{\alpha_{4}}, E_{3}=x_{\alpha_{3}}$,

$E_{4}=x_{\alpha_{6}} ; F_{0}=x_{\theta^{0}}-x_{\bar{\mu}\left(\theta^{0}\right)}, F_{1}=x_{-\alpha_{1}}+x_{-\alpha_{5}}, F_{2}=x_{-\alpha_{2}}+x_{-\alpha_{4}}$,

$F_{3}=x_{-\alpha_{3}}, F_{4}=x_{-\alpha_{6}} ; H_{i}=\left[E_{i}, F_{i}\right](0 \leq i \leq 4)$.

Case 5: $\mathfrak{g}=D_{4}, r=3, \varepsilon=\exp (2 \pi i / 3) ; \bar{\mu}\left(\alpha_{1}\right)=\alpha_{4}, \bar{\mu}\left(\alpha_{2}\right)=\alpha_{2}, \bar{\mu}\left(\alpha_{3}\right)=\alpha_{1}$,

$\bar{\mu}\left(\alpha_{4}\right)=\alpha_{3}, \mu\left(h_{\alpha}\right)=h_{\bar{\mu}(\alpha)}, \mu\left(x_{\alpha}\right)=x_{\bar{\mu}(\alpha)} ; \theta=\alpha_{1}+2 \alpha_{2}+\alpha_{3}+\alpha_{4}$,

$\theta^{0}=\theta-\alpha_{2}-\alpha_{4},-\beta_{0}=\left.\frac{1}{3}\left(\theta^{0}+\bar{\mu}\left(\theta^{0}\right)+\bar{\mu}^{2}\left(\theta^{0}\right)\right)\right|_{\mathfrak{t}_{[0]}}$,

$\beta_{1}=\left.\alpha_{1}\right|_{\mathfrak{t}_{[0]}}=\left.\alpha_{3}\right|_{\mathfrak{t}_{[0]}}=\left.\alpha_{4}\right|_{\mathfrak{t}_{[0]}}, \beta_{2}=\left.\alpha_{2}\right|_{\mathfrak{t}_{[0]}} ;$

$E_{0}=x_{-\theta^{0}}+\varepsilon^{2} x_{-\bar{\mu}\left(\theta^{0}\right)}+\varepsilon x_{-\bar{\mu}^{2}\left(\theta^{0}\right)}, E_{1}=x_{\alpha_{1}}+x_{\alpha_{3}}+x_{\alpha_{4}}, E_{2}=x_{\alpha_{2}} ;$

$F_{0}=x_{\theta^{0}}+\varepsilon x_{\bar{\mu}\left(\theta^{0}\right)}+\varepsilon^{2} x_{\bar{\mu}^{2}\left(\theta^{0}\right)}, F_{1}=x_{-\alpha_{1}}+x_{-\alpha_{3}}+x_{-\alpha_{4}}, F_{2}=x_{-\alpha_{2}}$;

$H_{i}=\left[E_{i}, F_{i}\right](0 \leq i \leq 2)$.

Note that $\mathfrak{g}$ is simply-laced and that $x_{\theta}$ is $\mu$-homogeneous in all these cases. The canonical generators $\left\{e_{i}, f_{i}, h_{i} \mid 0 \leq i \leq l\right\}$ of $\hat{\mathfrak{g}}[\mu]$ are as in (1.3), with $\nu=\mu$ and $\left(s_{0}, s_{1}, \ldots, s_{l}\right)=(1,0, \ldots, 0)$. Set now

$$
R=U(\mathfrak{g}) x_{\theta}(-1)^{k+1} \mathbf{1} \subset N\left(k \Lambda_{0}\right) .
$$

Clearly, $R$ is isomorphic to the simple $\mathfrak{g}$-module with highest weight $(k+1) \theta$. Notice also that $R$ is invariant under all automorphisms of the form (2.14) and recall from (1.4) the triangular decomposition of $\hat{\mathfrak{g}}$. The following result may be found in MP2, Corollary 5.4 \& Lemma 5.5]:

Proposition 2.7. The maximal submodule $N^{1}\left(k \Lambda_{0}\right)$ of $N\left(k \Lambda_{0}\right)$ is generated by the singular vector $x_{\theta}(-1)^{k+1} \mathbf{1}$. Hence

$$
N^{1}\left(k \Lambda_{0}\right)=U(\hat{\mathfrak{g}}) x_{\theta}(-1)^{k+1} \mathbf{1}=U\left(\mathfrak{n}_{-}\right) x_{\theta}(-1)^{k+1} \mathbf{1} .
$$

Let $M$ be a restricted $\tilde{\mathfrak{g}}[\mu]$-module of level $k$ and define $\bar{R}_{\mu}$ as in (2.1). Proposition 2.7 implies that $\mathfrak{g}(n) R=0$ for all integers $n \geq 1$, and then Theorem 2.1 yields

Corollary 2.8. $\bar{R}_{\mu}$ is an irreducible loop $\tilde{\mathfrak{g}}[\mu]$-module.

Recall from Theorem 1.2 that any restricted (in particular, any integrable) $\tilde{\mathfrak{g}}[\mu]$ module of level $k$ is a weak $\mu$-twisted $N\left(k \Lambda_{0}\right)$-module. We can now prove the following

Theorem 2.9. If $L(\Lambda)$ is a standard $\tilde{\mathfrak{g}}[\mu]$-module of level $k$ then $\bar{R}_{\mu} L(\Lambda)=0$.

Proof. Let $M=L(\Lambda)$. By the twisted associator formula $(1.10)$ and induction, it suffices to prove that $Y_{M}^{\mu}\left(x_{\theta}(-1)^{k+1} \mathbf{1}, z\right)=0$. Recall that $\left\langle x_{\theta}, x_{-\theta}\right\rangle=1$ and notice that $x_{\theta} \in \mathfrak{g}_{[1]}$ in case 1 , while $x_{\theta} \in \mathfrak{g}_{[0]}$ in cases 2-5. Let $\mathfrak{a}=\mathbf{C}$-span $\left\{x_{\theta}, x_{-\theta}, h_{\theta}\right\} \cong$ $\mathfrak{s l}(2, \mathbf{C}))$. Then we can embed $\widehat{\mathfrak{s l}(2, \mathbf{C})} \cong \hat{\mathfrak{a}}$ into $\hat{\mathfrak{g}}[\mu]$ with canonical central element $c$ in cases 2-5. Since $M$ is a level $k$ integrable $\hat{\mathfrak{g}}[\mu]$-module, it is $\grave{a}$ fortiori an integrable $\hat{\mathfrak{a}}$-module of level $k$. As such, $\left(M, Y_{M}^{\mu}\right)$ becomes by Theorem 1.1 an (untwisted) weak module for the $\operatorname{VOA} N\left(k \Lambda_{0} ; \hat{\mathfrak{a}}\right) \subset N\left(k \Lambda_{0}\right)$ via the restricted 
vertex map. It then follows from Proposition 2.6 that $Y_{M}^{\mu}\left(x_{\theta}(-1) \mathbf{1}, z\right)^{k+1}=0$. Since $\left[Y_{M}^{\mu}\left(x_{\theta}(-1) \mathbf{1}, z_{1}\right), Y_{M}^{\mu}\left(x_{\theta}(-1) \mathbf{1}, z_{2}\right)\right]=0$, one gets from (1.10) that

$$
Y_{M}^{\mu}\left(x_{\theta}(-1)^{k+1} \mathbf{1}, z\right)=Y_{M}^{\mu}\left(x_{\theta}(-1) \mathbf{1}, z\right)^{k+1}=0
$$

in cases $2-5$, as needed.

Note that in case 1 we can embed $\widehat{\mathfrak{s} \mathfrak{l}(2, \mathbf{C})}[\mu] \cong \hat{\mathfrak{a}}[\mu]$ into $\hat{\mathfrak{g}}[\mu]$ with canonical central element $c$ and that the restriction of $\mu$ to $N\left(k \Lambda_{0} ; \hat{\mathfrak{a}}\right)$ coincides with the inner automorphism $\exp \left(\pi i h_{\theta}(0) / 2\right)$. As above, $M$ is an integrable $\hat{\mathfrak{a}}[\mu]$-module of level $k$ and thus $\left(M, Y_{M}^{\mu}\right)$ becomes by Theorem 1.2 a weak $\mu$-twisted $N\left(k \Lambda_{0} ; \hat{\mathfrak{a}}\right)$ module via the restricted vertex map. Using Proposition 2.3 with $h=-h_{\theta} / 4$ and $\bar{Y}_{M}^{\mu}(\cdot, z)=Y_{M}^{\mu}(\Delta(h, z) \cdot, z)$, one gets that $\left(M, \bar{Y}_{M}^{\mu}\right)$ is an untwisted weak $N\left(k \Lambda_{0} ; \hat{\mathfrak{a}}\right)$-module. Consequently, $M$ acquires a structure of level $k$ integrable $\hat{\mathfrak{a}}$ module via the vertex map $\bar{Y}_{M}^{\mu}(\cdot, z)$. Moreover, it follows from $h_{\theta}(n) x_{\theta}(-1)^{p} \mathbf{1}=0$ for $n, p \in \mathbf{Z}_{>0}$ that $\Delta(h, z) x_{\theta}(-1)^{p} \mathbf{1}=z^{-p / 2} x_{\theta}(-1)^{p} \mathbf{1}$, which combined with (1.10) and (2.13) gives

$$
\begin{aligned}
\bar{Y}_{M}^{\mu}\left(x_{\theta}(-1)^{p} \mathbf{1}, z\right)=z^{-p / 2} Y_{M}^{\mu}\left(x_{\theta}(-1)^{p} \mathbf{1}, z\right) \\
\quad=z^{-p / 2} Y_{M}^{\mu}\left(x_{\theta}(-1) \mathbf{1}, z\right)^{p}=\bar{Y}_{M}^{\mu}\left(x_{\theta}(-1) \mathbf{1}, z\right)^{p}
\end{aligned}
$$

Proposition 2.6 again implies that $\bar{Y}_{M}^{\mu}\left(x_{\theta}(-1)^{k+1} \mathbf{1}, z\right)=\bar{Y}_{M}^{\mu}\left(x_{\theta}(-1) \mathbf{1}, z\right)^{k+1}=0$, and then 2.16) yields $Y_{M}^{\mu}\left(x_{\theta}(-1)^{k+1} \mathbf{1}, z\right)=0$. The proof is thereby complete.

Since $\mathfrak{g}$ is simply-laced in all the cases listed above, one gets from (2.15) and the action of the Weyl group of $\mathfrak{g}$ that

$$
x_{\alpha}(-1)^{k+1} \mathbf{1} \in R \text { for } \alpha \in \Phi .
$$

Dealing with case 1 requires the following

Lemma 2.10. Let $\mathfrak{l}$ be a 3-dimensional Heisenberg algebra with basis $\{x, y, z\}$ such that $[x, y]=z,[x, z]=[y, z]=0$. For every $m \in \mathbf{Z}_{\geq 0}$, the following holds in $U(\mathfrak{l})$ :

$$
(x+y)^{m} \in \sum_{\substack{a+b+2 c=m \\ a \leq b}} \mathbf{C} x^{a} y^{b} z^{c}+\sum_{\substack{a+b+2 c=m \\ b \leq a}} \mathbf{C} y^{b} x^{a} z^{c} .
$$

Proof. Let $\omega$ be the associative algebra automorphism of $U(\mathfrak{l})$ induced by the Lie algebra involution of $\mathfrak{l}$ defined by $\omega(x)=y, \omega(y)=x, \omega(z)=-z$. Let $a, b, c \in \mathbf{Z}_{\geq 0}$ be such that $a \leq b$ and $a+b+2 c=n$, where $n \in \mathbf{Z}_{\geq 0}$ is arbitrarily fixed. An easy induction gives

$$
x y^{p}=y^{p} x+p y^{p-1} z, \quad y x^{p}=x^{p} y-p x^{p-1} z
$$

for $p \geq 1$, so that if $a<b$ it follows that

$$
x^{a} y^{b} z^{c} x \in \sum_{\substack{\alpha+\beta+2 \gamma=n+1 \\ \alpha \leq \beta}} \mathbf{C} x^{\alpha} y^{\beta} z^{\gamma} .
$$

Suppose now that $a=b$. Using (2.19) and induction one gets that $x^{p} y^{p} \in$ $\sum_{\alpha+\gamma=n} \mathbf{C} y^{\alpha} x^{\alpha} z^{\gamma}$ whenever $p \geq 1$, and therefore

$$
x^{a} y^{a} z^{c} x \in \sum_{\substack{\alpha+\beta+2 \gamma=n+1 \\ \beta \leq \alpha}} \mathbf{C} y^{\beta} x^{\alpha} z^{\gamma} .
$$


It follows from $(2.20)$ and $(2.21)$ that in both cases

$$
x^{a} y^{b} z^{c} x \in \sum_{\substack{\alpha+\beta+2 \gamma=n+1 \\ \alpha \leq \beta}} \mathbf{C} x^{\alpha} y^{\beta} z^{\gamma}+\sum_{\substack{\alpha+\beta+2 \gamma=n+1 \\ \beta \leq \alpha}} \mathbf{C} y^{\beta} x^{\alpha} z^{\gamma} .
$$

Consequently

$$
y^{a} x^{b} z^{c} y \in \sum_{\substack{\alpha+\beta+2 \gamma=n+1 \\ \alpha \leq \beta}} \mathbf{C} y^{\alpha} x^{\beta} z^{\gamma}+\sum_{\substack{\alpha+\beta+2 \gamma=n+1 \\ \beta \leq \alpha}} \mathbf{C} x^{\beta} y^{\alpha} z^{\gamma}
$$

by using (2.22) and the action of $\omega$. Then (2.18) follows by induction on $m$ from $(x+y)^{m}=(x+y)^{m-1}(x+y)$ together with (2.22) and (2.23).

Proposition 2.11. Let $\mathfrak{g}$ be as above. Then $F_{i}(-1)^{n} \mathbf{1} \in N^{1}\left(k \Lambda_{0}\right)$ for all $i \in$ $\{0,1, \ldots, l\}$ if $n$ is sufficiently large.

Proof. Using the explicit form of the root systems $\Phi$, it is readily checked that in cases $2-5$ the $F_{i}$ 's are sums of at most three commuting root vectors of $\mathfrak{g}$. Therefore

$$
F_{i}(-1)^{t k+1} \mathbf{1} \in N^{1}\left(k \Lambda_{0}\right), \quad i \in\{0,1, \ldots, l\},
$$

for some $t \in\{1,2,3\}$ by (2.17). The same argument implies that in case 1

$$
F_{i}(-1)^{2 k+1} \mathbf{1} \in N^{1}\left(k \Lambda_{0}\right), \quad i \in\{1, \ldots, l-1\},
$$

while obviously $F_{0}(-1)^{k+1} \mathbf{1}=x_{\theta}(-1)^{k+1} \mathbf{1} \in N^{1}\left(k \Lambda_{0}\right)$. Applying now Lemma 2.10 with $x=x_{-\alpha_{l}}(-1), y=x_{-\alpha_{l+1}}(-1)$, and $z=\left[x_{-\alpha_{l}}(-1), x_{-\alpha_{l+1}}(-1)\right]$ one gets $F_{l}(-1)^{4 k+1} \mathbf{1} \in N^{1}\left(k \Lambda_{0}\right)$, so that the proposition is true in all cases.

We are now ready to prove a more complete version of Theorem 2.9:

Theorem 2.12. Let $M(\Lambda)$ denote the Verma $\hat{\mathfrak{g}}[\mu]$-module with highest weight $\Lambda \in$ $P_{+}$such that $\Lambda(c)=k$. Then $\bar{R}_{\mu} M(\Lambda)=M^{1}(\Lambda)$, where $M^{1}(\Lambda)$ is the maximal submodule of $M(\Lambda)$.

Proof. Let $v_{\Lambda}$ be a highest weight vector of $M:=M(\Lambda)$ and set $V=\bar{R}_{\mu} M(\Lambda)$. By Theorem 2.9 it suffices to prove that $M^{1}(\Lambda) \subset V$, which in turn reduces to showing that

$$
\left\{f_{i}^{\Lambda\left(h_{i}\right)+1} v_{\Lambda} \mid i=0,1, \ldots, l\right\} \subset V
$$

by (1.6). According to Propositions 2.11 and $2.7, F_{i}(-1)^{t k+1} \mathbf{1} \in U(\hat{\mathfrak{g}}) x_{\theta}(-1)^{k+1} \mathbf{1}=$ $N^{1}\left(k \Lambda_{0}\right)$ for some $t \in\{1,2,3,4\}$. It then follows from (1.10), (2.6) and induction that

$$
Y_{M}^{\mu}\left(F_{i}(-1)^{t k+1} \mathbf{1}, z\right) v_{\Lambda} \in V\left[\left[z^{1 / r}, z^{-1 / r}\right]\right]
$$

and consequently

$$
Y_{M}^{\mu}\left(F_{i}(-1) \mathbf{1}, z\right)^{t k+1} v_{\Lambda} \in V\left[\left[z^{1 / r}, z^{-1 / r}\right]\right]
$$

(since $\left[Y_{M}^{\mu}\left(F_{i}(-1) \mathbf{1}, z_{1}\right), Y_{M}^{\mu}\left(F_{i}(-1) \mathbf{1}, z_{2}\right)\right]=0$ for $\left.i=0,1, \ldots, l\right)$. Hence

$$
f_{i}^{t k+1} v_{\Lambda}=\operatorname{Res}_{z} z^{t k} Y_{M}^{\mu}\left(F_{i}(-1) \mathbf{1}, z\right)^{t k+1} v_{\Lambda} \in V
$$

if $i \in\{1, \ldots, l\}$, and

$$
f_{0}^{t k+1} v_{\Lambda}=\operatorname{Res}_{z} z^{\left(1-\frac{1}{r}\right)(t k+1)-1} Y_{M}^{\mu}\left(F_{0}(-1) \mathbf{1}, z\right)^{t k+1} v_{\Lambda} \in V,
$$


where $t=1,2,3$ or 4 according to Proposition 2.11. Since $\left[e_{i}, f_{i}\right]=h_{i}$ and $h_{i} v_{\Lambda}=$ $\Lambda\left(h_{i}\right) v_{\Lambda}$, the representation theory of $\mathfrak{s l}(2, \mathbf{C})$ together with $(2.6),(2.24)$ and $(2.25)$ imply that

$$
f_{i}^{\Lambda\left(h_{i}\right)+1} v_{\Lambda} \subset V, \quad i=0,1, \ldots, l
$$

as required.

It is easy to see that only some minor changes are needed in order to make the above arguments also work in the case of the trivial diagram automorphism id $\mathrm{id}_{\mathfrak{g}}$. In fact, for the trivial twist most of these arguments can be simplified and one obtains in this way the corresponding theorems in the untwisted case (cf. [MP2, Theorems $5.9 \& 5.14]$ ). Then one can extend the previous results to arbitrary finite-order automorphisms, as shown by the following

Theorem 2.13. Let $\sigma, \psi \in \operatorname{Aut}(\mathfrak{g})$ be such that $\sigma \psi=\psi \mu \exp \left(\operatorname{ad}\left(\frac{2 \pi i}{T} h\right)\right)$ for some appropriate $h \in \mathfrak{t}_{[0]}$ and some diagram automorphism $\mu$ defined with respect to $(\mathfrak{t}, \Pi)$, where $T$ is the order of $\sigma$. Let $M^{1}(\Lambda)$ be the maximal submodule of the level $k$ Verma $\hat{\mathfrak{g}}[\sigma]$-module $M(\Lambda)$ with highest weight $\Lambda \in P_{+}$. Then

$$
\overline{\psi(R)_{\sigma}} M(\Lambda)=M^{1}(\Lambda),
$$

where $R$ is as in (2.15) and $\overline{\psi(R)}_{\sigma}=\mathbf{C}$-span $\left\{\operatorname{Res}_{z} z^{n} Y_{M(\Lambda)}^{\sigma}(r, z) \mid r \in \psi(R), n \in\right.$ $\left.\frac{1}{T} \mathbf{Z}\right\}$.

Proof. Note first that by Proposition 2.5 there exist indeed $\psi \in \operatorname{Aut}(\mathfrak{g})$ and $h \in \mathfrak{t}_{[0]}$ as specified in the assumptions. Set $\mathfrak{t}^{\prime}=\psi(\mathfrak{t}), \mu^{\prime}=\psi \mu \psi^{-1}, h^{\prime}=\psi(h), R^{\prime}=\psi(R)$, and recall the automorphism $\bar{\mu}$ of order $r$ of the Dynkin diagram of $\mathfrak{g}$. Then $\mathfrak{t}^{\prime}$ is a $C S A$ of $\mathfrak{g}$ and $\mu^{\prime}$ is the diagram automorphism of $\mathfrak{g}$ induced by the automorphism $\bar{\mu}$ with respect to $\left(\mathfrak{t}^{\prime}, \Pi\right)$. Let $\mathfrak{t}^{\prime}=\coprod_{j \in \mathbf{Z}_{r}} \mathfrak{t}_{[j]}^{\prime}$ be the $\mu^{\prime}$-decomposition of $\mathfrak{t}^{\prime}$ and notice that $h^{\prime} \in \mathfrak{t}_{[0]}^{\prime}$ and that $\sigma=\mu^{\prime} \exp \left(\operatorname{ad}\left(\frac{2 \pi i}{T} h^{\prime}\right)\right)$. Moreover, $R^{\prime}$ is $\exp \left(\operatorname{ad}\left(\frac{2 \pi i}{T} h^{\prime}\right)\right)$-invariant and it obviously satisfies $\mathfrak{g}(n) R^{\prime}=0$ for all integers $n \geq 1$. Then (2.26) follows by combining Proposition 2.3 with Theorem 2.12 applied to the data $\left(\mathfrak{t}^{\prime}, \Pi, R^{\prime}, \mu^{\prime}\right)$.

We now obtain the following characterization of standard modules in terms of (actions of) irreducible loop $\tilde{\mathfrak{g}}[\sigma]$-modules:

Theorem 2.14. Let $\sigma, \psi \in \operatorname{Aut}(\mathfrak{g})$ be as in Theorem 2.13, and let $M$ be a highest weight $\hat{\mathfrak{g}}[\sigma]$-module of level $k$. Then $M$ is a standard module if and only if $\overline{\psi(R)_{\sigma}}$ annihilates $M$.

Proof. By Theorem 2.13 it suffices to consider only the case when $\sigma=\mu$ and $\psi=\operatorname{id}_{\mathfrak{g}}$. Let $\Lambda$ be the highest weight of $\mathrm{M}$ and let $v_{\Lambda} \in M$ be a highest weight vector. If $\bar{R}_{\mu} M=0$, then one can argue as in the proof of Theorem 2.12 to show that for $0 \leq i \leq l$ one has $f_{i}^{t k+1} v_{\Lambda} \in \bar{R}_{\mu} M=0$ for some $t \in\{1,2,3,4\}$, so that $M$ is necessarily integrable. The converse is given by Theorem 2.9 . 


\section{REFERENCES}

[Ad] D. Adamović, Some rational vertex algebras, Glas. Mat. Ser. 29 (1994), 25-40.

[An] G. E. Andrews, The theory of partitions, Addison-Wesley, Amsterdam, 1976.

[Bo] J. Borcea, Dualities and affine vertex operator algebras, preprint (1999).

[B] R. E. Borcherds, Vertex algebras, Kac-Moody algebras, and the Monster, Proc. Natl. Acad. Sci. USA 83 (1986), 3068-3071.

[Ca1] S. Capparelli, Elements of the annihilating ideal of a standard module, J. Algebra 145 (1992), 32-54.

[Ca2] On some representations of twisted affine Lie algebras and combinatorial identities, J. Algebra 154 (1993), 335-355.

[Ca3] A construction of the level 3 modules for the affine Lie algebra $A_{2}^{(2)}$ and a new combinatorial identity of the Rogers-Ramanujan type, Trans. Amer. Math. Soc. 348 (1996), 481-501.

[CP] V. Chari, A. Pressley, Integrable Representations of Kac-Moody Algebras: Results and Open Problems, in Infinite-Dimensional Lie Algebras and Groups, Adv. Ser. Math. Phys. Vol. 7 (V. G. Kac, ed.), World Scientific, Singapore, 1989.

[DL] C. Dong, J. Lepowsky, Generalized vertex algebras and relative vertex operators, Progr. Math. 112, Birkhäuser, Boston, 1993.

[FHL] I. B. Frenkel, Y.-Z. Huang, J. Lepowsky, On axiomatic approaches to vertex operator algebras and modules, Mem. Amer. Math. Soc. 494, 1993.

[FLM] I. B. Frenkel, J. Lepowsky, A. Meurman, Vertex Operator Algebras and the Monster, Pure Appl. Math. 134, Academic Press, New York, 1988.

[FM] I. B. Frenkel, F. Malikov, Annihilating ideals and tilting functors, preprint math.QA/9801065.

[K] V. Kac, Infinite-dimensional Lie Algebras, 3rd ed., Cambridge Univ. Press, Cambridge, 1990.

[KKLW] V. Kac, D. A. Kazhdan, J. Lepowsky, R. L. Wilson, Realization of the Basic Representations of the Euclidean Lie Algebras, Adv. Math. 42 (1981), 83-112.

$[\mathrm{KW}] \quad$ V. Kac, M. Wakimoto, Classification of Modular Invariant Representations of Affine Lie Algebras, in Infinite-Dimensional Lie Algebras and Groups, Adv. Ser. Math. Phys. Vol. 7 (V. G. Kac, ed.), World Scientific, Singapore, 1989.

[LM] J. Lepowsky, S. Milne, Lie Algebraic Approaches to Classical Partition Identities, Adv. Math. 29 (1978), 15-59.

[LP] J. Lepowsky, M. Primc, Structure of standard modules for the affine Lie algebra $A_{1}^{(1)}$, Contemp. Math. 46, 1985.

[LW1] J. Lepowsky, R. L. Wilson, A new family of algebras underlying the Rogers-Ramanujan identities and generalizations, Proc. Natl. Acad. Sci. USA 78 (1981), 7254-7258.

[LW2] - The structure of standard modules, I: Universal algebras and the RogersRamanujan identities, Invent. Math. 77 (1984), 199-290; II: The case $A_{1}^{(1)}$, principal gradation, Invent. Math. 79 (1985), 417-442.

[Li1] H.-S. Li, Local Systems of Vertex Operators, Vertex Superalgebras and Modules, J. Pure Appl. Algebra 109 (1996), 143-195.

[Li2] Local systems of twisted vertex operators, vertex operator superalgebras and twisted modules, Contemp. Math. 193 (1996), 203-236.

[MP1] A. Meurman, M. Primc, Annihilating ideals of standard modules of $\mathfrak{s l}(2, \mathbf{C})^{r}$ and combinatorial identities, Adv. Math. 64 (1987), 177-240.

[MP2] Annihilating fields of standard modules of $\mathfrak{s l}(2, \mathbf{C})^{\sim}$ and combinatorial identities, Mem. Amer. Math. Soc. 652, 1999.

[MP3] , A basis of the basic $\mathfrak{s l}(3, \mathbf{C})$ - module, preprint math.QA/9812029.

[P1] M. Primc, Loop modules in annihilating ideals of standard modules for affine Lie algebras, Grazer Math. Ber. 313 (1991), 39-44.

[P2] Vertex operator constructions of standard modules for $A_{n}^{(1)}$, Pacific J. Math. 162 (1994), 143-187.

[TX] M. Tamba, C.-F. Xie, Level three standard modules for $A_{2}^{(2)}$ and combinatorial identities, J. Pure Appl. Algebra 105 (1995), 53-92. 
Institut de Recherche Mathématique Avancée, Université Louis Pasteur, 7 Rue René Descartes, 67084 Strasbourg Cedex, France

E-mail address: borcea@math.u-strasbg.fr 